\title{
On-line 3D surface measurement of iron ore green pellets
}

\author{
Matthew J Thurley \\ Luleå University of Technology \\ Luleå, Sweden \\ matthew.thurley@1tu.se \\ www.csee.ltu.se/ mjt
}

\begin{abstract}
Ongoing research into an industrial prototype $3 \mathrm{D}$ imaging and analysis system is presented for non-contact measurement of the size of iron ore green pellets on the conveyor belt. The imaging hardware has been installed at a pellet production plant and captures 3D surface data of pellets on the conveyor belt. Segmentation methods based on mathematical morphology are applied to the $3 D$ surface data to identify individual pellets. Pellet sizing based on the segmented 3D surface data has been performed and demonstrates a correlation with pellet sieve size.
\end{abstract}

\section{Introduction}

In any manufacturing process quality control is a vital concern. For iron ore pellet producers adherence to the target green pellet size range is one of the major measures of quality. Green pellets are produced primarily from crushed iron ore in a rotating pelletising disk or tumbling drum after which they are baked in a furnace to produce hardened black pellets.

Existing measurement of green pellet size is a manual sampling and sieving process which is invasive, infrequent, and overly time consuming preventing rapid response to size variation. Ongoing research and implementation of an industrial prototype 3D imaging and analysis system is presented for non-contact measurement of the size of green pellets on the conveyor belt. The advantages are frequent sampling and fast generation of sizing results providing rapid feedback to the manufacturing process.

An imaging system that captures 3D surface data has been implemented by MBV-systems [5]. The system is based on a projected laser line and camera triangulation [1, triangulation, structured light]. It has a high speed digital camera capable of 4000 frames per second and a continuous wave diode laser with line generating optics. The angle between the camera line of sight and the laser is approximately
30 degrees. The imaging system is installed and operational at a local pellet plant collecting 3D surface coordinate data at an irregular density of approximately $0.5 \mathrm{~mm}^{2}$.

$3 \mathrm{D}$ surface data analysis software based on the work of Thurley [6] is being applied to the segmentation and sizing of the data. The current results of this analysis are presented with the final segmentation and sizing solution to be completed by December 2006.

\section{Collection of 3D Surface Data}

The data collected by this system comprises irregularly spaced 3D coordinate points describing the surface of the pellet pile on the conveyor belt. Data covering approximately one meter of the belt is collected and then analysed. After analysis is completed the process is repeated. Figure 1 shows an enhanced photograph of the laser line on the moving conveyor pellet pile and figure 3 on page 3 shows a portion of the $3 \mathrm{D}$ surface of a pellet pile.

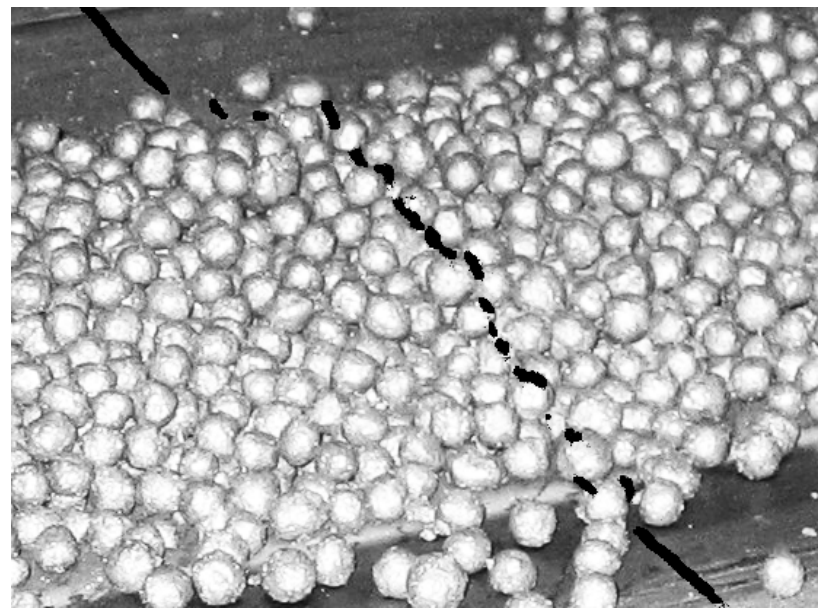

Figure 1. Laser line across green pellets (enhanced image) 


\section{Analysis of 3D Surface Data}

\subsection{Overview}

There are some inherent complications when it comes to processing 3D surface data collected by laser triangulation, as follows;

1. There are numerous locations where the laser line is not visible from the point of view of the camera. This results in sparse areas in the 3D surface where there are no data points.

2. The data is irregularly spaced in the $(x, y)$ coordinate plane. That is, it is not defined in a regular image space such as a greyscale range image generated by a scanning laser range finder.

As a result of these two features of the data we cannot apply image based mathematical morphology operations without first resampling to a regular grid and interpolating to fill in sparse areas. Specifically, we cannot use the mathematical morphological operations in Matlab or any other image based mathematical morphological implementation on our raw data.

We note that mathematical morphology is a powerful image processing technique for analysing geometric structure with particular relevance to this problem. Mathematical morphology has applications in edge detection, enhancement, segmentation, reconstruction, texture analysis, particle analysis and shape analysis to name a few areas. The reader is referred to the book Hands-on Morphological Image Processing [3] for an extremely accessible and applied text on mathematical morphology and its applications.

However, resampling and interpolating the raw data to an image space is not desirable as it will introduce erroneous artifacts [8] into the data resulting in bias in the analysis.

We therefore present a segmentation and analysis solution based on a mathematical morphology implementation $[6,9]$ for sparse, irregularly spaced 3D surface data. As shown by Thurley and $\mathrm{Ng}[6,9]$ the application of this implementation can identify individual rocks on the surface of laboratory rock piles with sufficient accuracy to estimate the pile size distribution. Therefore, we shall use this mathematical morphology implementation as a framework for segmenting pellets.

\subsection{Segmentation Method}

1. Edge Detection 1: Find underlapping edges and remove underlapping data.

As a feature of the laser triangulation data capture system, some $(\mathrm{x}, \mathrm{y}, \mathrm{z})$ coordinate points are underlapping along the $\mathrm{z}$ axis. This occurs at the center facing edge of some pellets that are typically located out from the center of the pile. We use this underlapping information to find a few pellet edges and then we remove the underlapping data as it is not suitable for mathematical morphology.

\section{Edge Detection 2: Find edges of occluded areas}

Sparse or occluded areas where there are no data points occur at the edges of pellets. By detecting discontinuities in the column pixel count from the camera we detect some edges of occluded areas.

3. Noise Filtering: Median filtering in a $0.8 \mathrm{~mm}$ radius

4. Edge Detection 3: Morphological laplacian

This method does a good job of detecting the edge between overlapping pellets. We performed a morphological laplacian using a $0.8 \mathrm{~mm}$ radius spherical structuring element, take the absolute $\mathrm{z}$ value, perform a $0.8 \mathrm{~mm}$ spherical closing to fill gaps, and a $10 \%$ threshold on $\mathrm{z}$ depth.

\section{Edge Detection 4: Morphological black top hat}

This method is a good general edge detector. We performed a black top hat operation using a $1.6 \mathrm{~mm}$ radius spherical structuring element, followed by a $1.5 \%$ threshold on $\mathrm{z}$ depth.

\section{Edge Detection 5: Neighbourhood count}

We detect edges of sparse areas in the data by calculating the neighbour count for all data points using a $0.8 \mathrm{~mm}$ radius. Points with a low number of neighbours in the specified radius are likely edges of sparse areas in the data. We applied a threshold to retain only the smallest $40 \%$ of values from the neighbour count operation.

7. Combine Edges: Add all 5 edge detection methods together. Figure 2 shows a portion of the results of this operation.

8. Distance Transform on the combined edge set.

For each point we calculate the distance to the nearest edge point.

\section{Find Local Maxima of the Distance Transform}

Local maxima are determined using a morphological process called opening by reconstruction $[9$, section A.4] [3, pp. 136].

10. Seed Growing [6, 9] from Local Maxima 


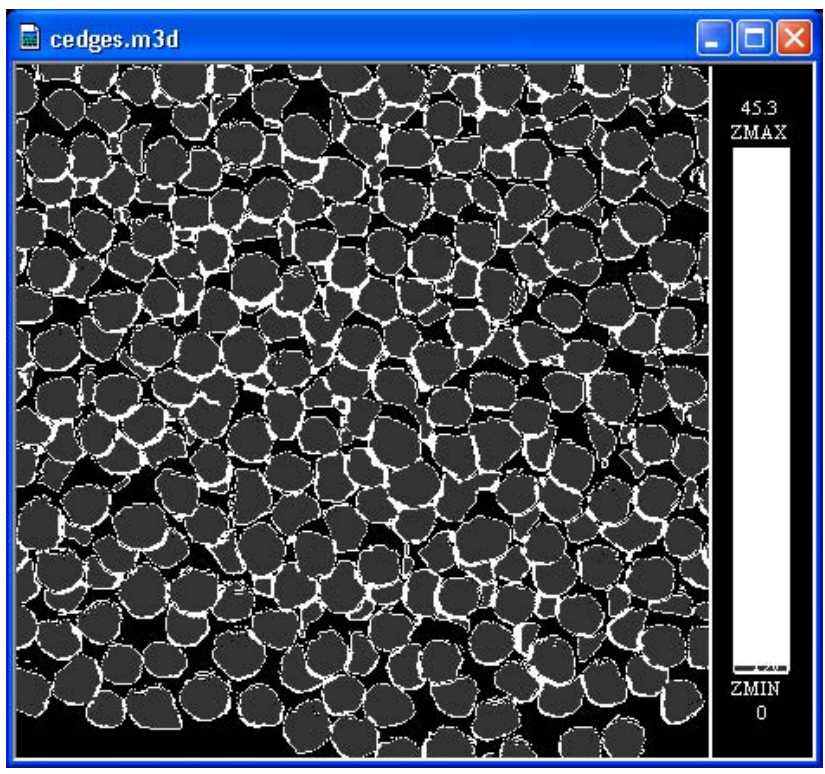

Figure 2. A portion of the detected pellet edges combining 5 techniques

11. Watershed Segmentation [3, ch. 7] [2]

A seeded watershed segmentation is performed to identify the individual pellet regions. This is a well established segmentation technique which is typically performed on the gradient representation of the input data. In this case we use the black top hat edge set as the detected region boundaries are more accurate for this data than when the morphological gradient edge set is used.

12. Post-filtering to remove tiny regions.

\subsection{Results}

A segmentation of a pile of baked pellets collected in the laboratory is presented. The data capture system in the laboratory is equivalent to that in the field, the only significant difference being that baked pellets have been used as green pellets are prohibitively fragile.

The segmentation identifies 1092 regions. After a region by region $3 \mathrm{D}$ visual analysis of the segmentation, with the segmentation data overlayed on the 3D surface we provide the following qualitative assessment.

- Over-segmentation occurred in 5 pellets that were reported at 10 regions.

- Under-segmentation occurred in 34 pellets that were reported as 17 regions.

- A segmentation shift occurred in 4 pellets that were reported as 4 regions but the region was shifted signif-

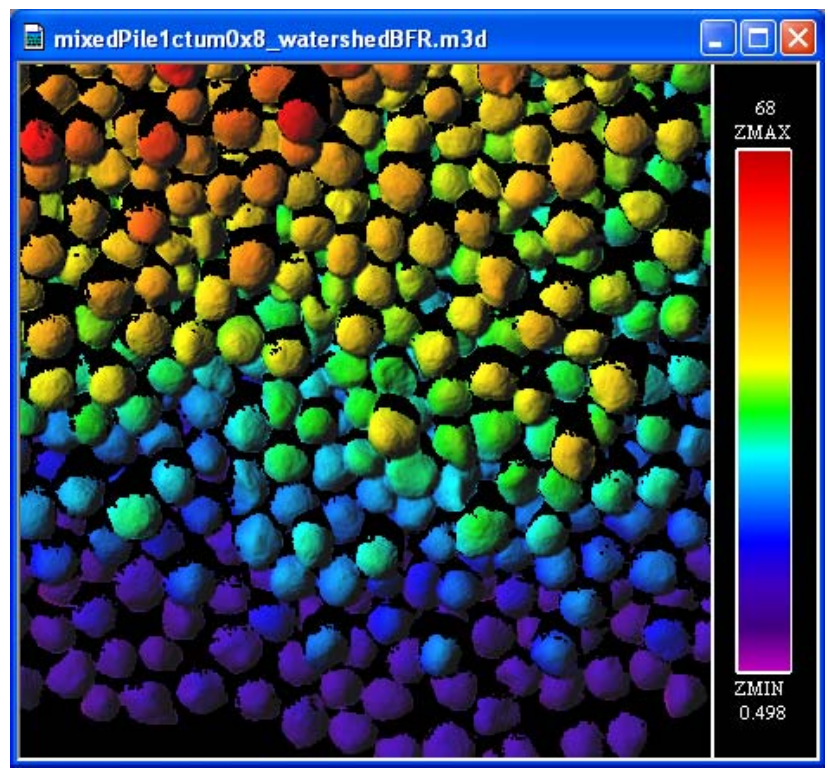

Figure 3. 3D surface data of a portion of the pellet pile

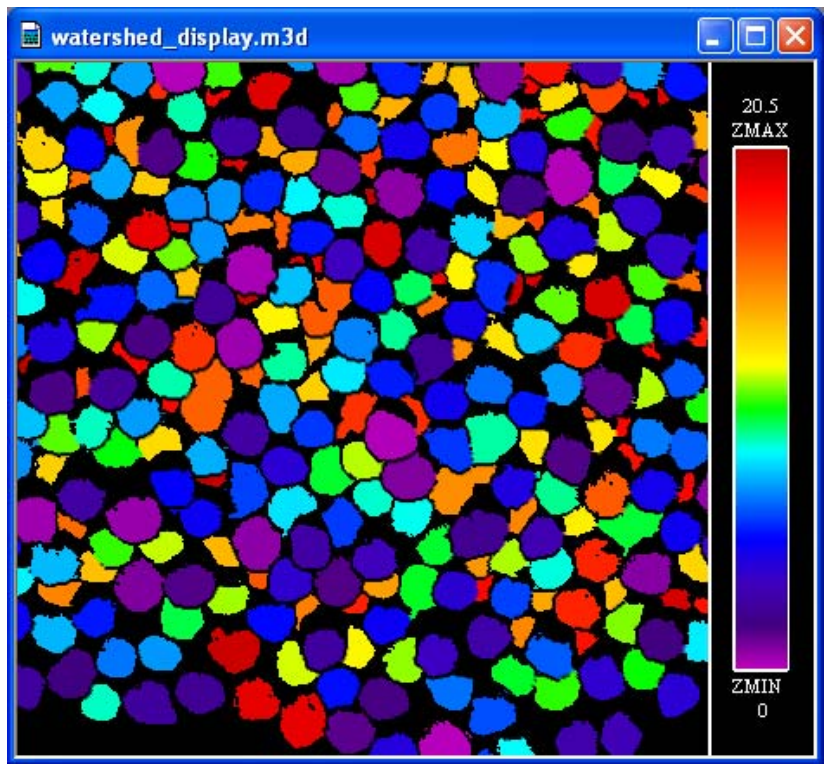

Figure 4. Segmentation of a portion of the pellet pile

icantly off the pellet. These regions corresponded to partially overlapped pellets.

- The 1092 detected regions represented 1104 pellets.

\subsection{Ongoing Research}

Before we can size the pellets detected by the segmentation we must make a determination of the pellets surface 
visibility for each region. That is, we must determine if a pellet is entirely visible, or it is overlapped by other pellets and is only partially visible.

This distinction between entirely and partially visible rock fragments has been performed for laboratory rock piles by Thurley and $\mathrm{Ng}[7,9]$. This distinction is very powerful as it allows accurate size determination of entirely visible rocks and for partially visible rocks to be either ignored or handled probabilistically. It is this distinction that most separates segmentation and sizing of 3D surface data from segmentation and sizing using 2D photographic methods.

However, the data presented in this research contains numerous sparse areas without data points and this condition is not handled by the published algorithm for rock visibility [7].

We note that as pellets as consistently blob like in shape, and not at all angular, it may be possible to use region shape as an indicator of pellet visibility. Entirely visible pellets should have regions that are roughly oval shaped when projected into the $x-y$ plane, whereas partially visible pellets should have regions with odd, convex and/or angular 2D shapes. Further work is in progress.

\section{Pellet Sizing}

Even though pellet sizing of the pile segmentation is not yet achieved, investigation of a sizing technique has been performed and compared against sieve results.

A $32 \mathrm{~kg}$ pellet sample was sieved into fine size gradings as shown in table 1 . In separate tests, a portion of each size fraction from $6.3 \mathrm{~mm}$ to $14 \mathrm{~mm}$ inclusive, was spread out on the laboratory conveyor belt in a single layer and scanned. Segmentation and sizing was then performed in order to calculate pellet size in optimal conditions (one size class with the surface of all pellets being entirely visible). The size classes $0-5 \mathrm{~mm}$ and $5-6.3 \mathrm{~mm}$ comprise only of small broken pellet pieces and are not indicative of green pellets. Therefore these size classes have been removed from the analysis. In addition the $16+\mathrm{mm}$ size class comprised of only 6 pellets and was removed from the analysis because it was not statistically significant.

We define region size by using a measure of area calculated from the encompassing best-fit-rectangle [10] of a region. This method has been used to size rock fragments in laboratory rock piles from 3D surface data [9].

The best-fit-rectangle is calculated in the $x-y$ plane projection of each region. We present the distribution of region area for each size class in table 2 and in figure 5 using the graphical convention of horizontal box-plots.

The central portion of a box-plot contains a rectangular box. In the center of this box is a short thick vertical black line, this marks the median value (or 50 percentile) of the data. The left (or lower) edge of the rectangular box

\begin{tabular}{|cccc|}
\hline Sieve Size $\dagger(\mathbf{m m})$ & Weight $\mathbf{( k g )}$ & \% & Cum. \% \\
\hline 0 & 0.264 & 0.8 & 0.8 \\
5 & 0.1098 & 0.3 & 1.2 \\
$\mathbf{6 . 3}$ & 4.089 & 12.7 & 13.8 \\
$\mathbf{9}$ & 4.755 & 14.7 & 28.6 \\
$\mathbf{1 0}$ & 12.613 & 39.1 & 67.7 \\
$\mathbf{1 1 . 2}$ & 7.493 & 23.2 & 90.9 \\
$\mathbf{1 2 . 5}$ & 2.445 & 7.6 & 98.5 \\
$\mathbf{1 4}$ & 0.4233 & 1.3 & 99.8 \\
$16+$ & 0.0613 & 0.2 & 100 \\
\hline Total Weight & 32.253 & & \\
\hline & The specified size is the lower bound of each sieve \\
size increment & & \\
\end{tabular}

\section{Table 1. Sieve Size distribution of the sample of baked pellets}

marks the 25 percentile, and the right (or upper) edge of the box marks the 75 percentile. The circular dots to the left and right of each box-plot indicate values that are statistically determined to be outliers. They are too low or two high to be statistically valid and predominantly correspond to erroneous regions in the segmentation. The dashed lines extending to the left and right of the rectangular box extend to the statistically valid min and max. The graphs and determination of outliers were calculated using the $\mathrm{R}$ statistical analysis package [4].

\begin{tabular}{|cccccc|}
\hline Size & \multicolumn{6}{c}{ Region Area* } & $\left(\mathbf{m m}^{2}\right)$ & Reg. $\dagger$ & Outl. $\neq$ \\
$(\mathbf{m m})$ & 25th\%* & Median & 75th\% & & \\
\hline 6.3 & 66.97 & 76.74 & 87.04 & 1010 & 8 \\
9 & 88.14 & 95.69 & 104.4 & 755 & 19 \\
10 & 102.0 & 110.9 & 121.2 & 867 & 19 \\
11.2 & 128.8 & 141.7 & 155.6 & 677 & 5 \\
12.5 & 161.8 & 175.9 & 192.4 & 477 & 15 \\
14 & 188.8 & 209.7 & 227.0 & 61 & 0 \\
\hline
\end{tabular}

* Area of the encompassing best-fit-rectangle

$\star 25$ th percentile. $25 \%$ of the data less than this value

$\diamond 75$ th percentile. $75 \%$ of the data less than this value

$\dagger$ Number of regions processed

$\ddagger$ Number of statistical outliers found

\section{Table 2. Distribution of region area $\left(\mathrm{mm}^{2}\right)$ by size class for pellets in a single layer}

We observe from figure 5 and table 2 that the central portion (between the $25^{t h}$ and $75^{t h}$ percentiles) of the region area distribution at each size class is almost entirely separate from the other size classes. This is very encouraging particularly given the closeness of the sieve size classes we 


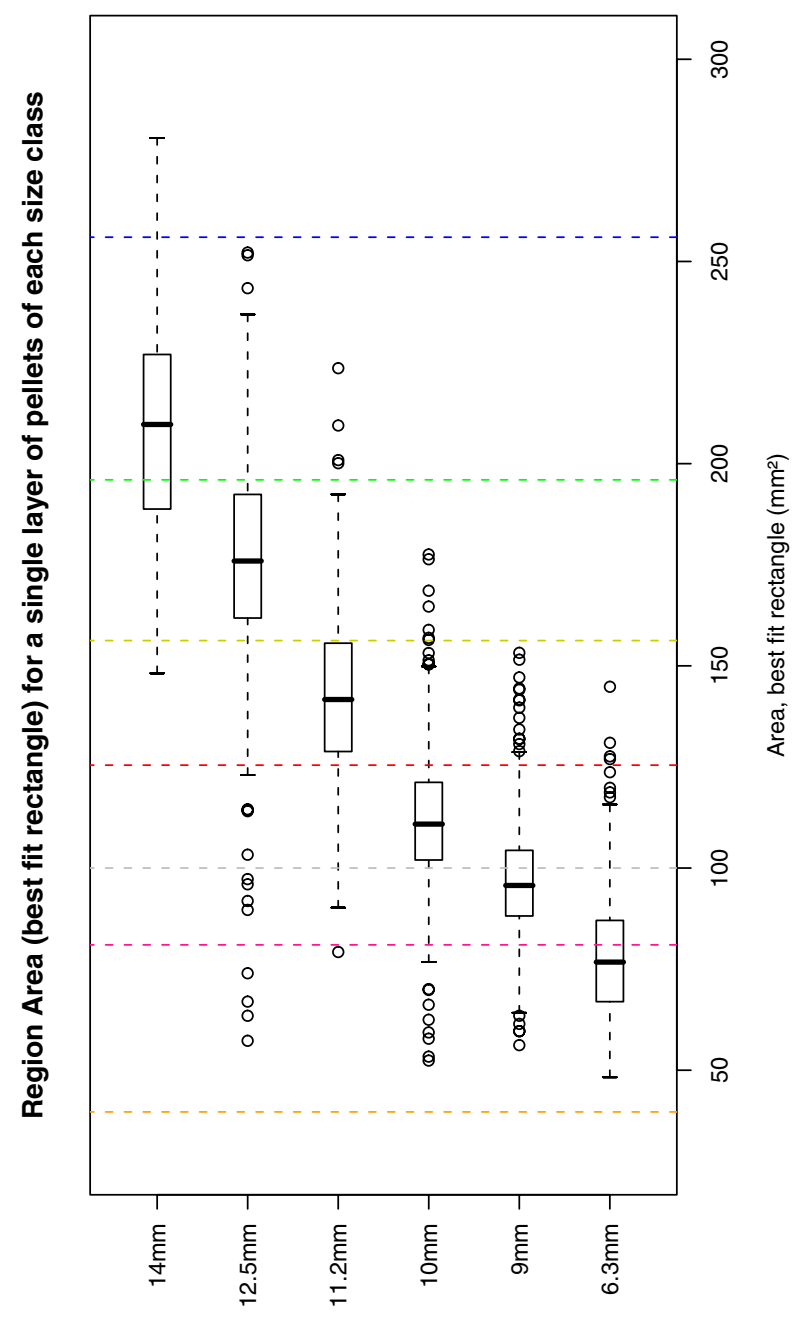

Figure 5. Distribution of region area by size class for pellets in a single layer.

are examining, being only of the order of $1 \mathrm{~mm}$ for several of the size classes.

Figure 5 shows a number of vertical dashed lines. These lines correspond to the area of the sieve meshes used to size the pellets, $39.69 \mathrm{~mm}^{2}$ for the $6.3 \mathrm{~mm}$ mesh, $81 \mathrm{~mm}^{2}$ for $9 \mathrm{~mm}, 100 \mathrm{~mm}^{2}$ for $10 \mathrm{~mm}, 125.44 \mathrm{~mm}^{2}$ for $11.2 \mathrm{~mm}, 156.25 \mathrm{~mm}^{2}$ for $12.5 \mathrm{~mm}, 196 \mathrm{~mm}^{2}$ for $14 \mathrm{~mm}$, and $256 \mathrm{~mm}^{2}$ for the $16 \mathrm{~mm}$ mesh. We note that the central portion of the region area distribution at each size class is predominantly contained within the corresponding sieve size range shown by the vertical dashed lines.

Based on the observed results and the success of this technique for sizing laboratory rock piles [7, 9] we expect that a classifier based on best-fit-rectangle area will be suf- ficient to classify pellets into sieve size classes in the industrial prototype system.

We recognise that best-fit-rectangle is a $2 \mathrm{D}$ measure of size which may seem limiting given we have measured 3D surface data. However, as noted above, best-fit-rectangle has been successfully applied to laboratory rock piles [9] and it shows promising results here. Initial investigations into 3D size measurement using sphere fitting have been unsatisfactory. Further research work into complimentary size measurement features is ongoing.

\section{Conclusion}

Ongoing research into a 3D imaging and analysis system for the online identification and sizing of iron ore green pellets on conveyor belt has been presented. A 3D surface data capturing system has been developed and installed at a local pellet plant. Individual pellets in the 3D surface data of the pellet pile have been identified using segmentation algorithms based on mathematical morphology for sparse, irregular 3D surface data. Furthermore, a sizing technique based on best-fit-rectangle shows very promising results for sizing pellets. A final segmentation and sizing solution is expected to be completed by December 2006. Research continues into complimentary size measurement features, and distinguishing between entirely and partially visible pellets on the pile surface.

\section{Acknowledgments}

Thank you to Olov Marklund, Sven Molin, Kim Ng, and the staff at ProcessIT Innovations for all their efforts in arranging and facilitating this opportunity to work in Sweden and apply my postgraduate research in industry. It is greatly appreciated. Thank you to John Erik Larsson at MBVsystems for his tireless efforts on the 3D surface imaging system, particularly to my demands for additional hardware capabilities in the laboratory system. Thank you to Tobias Andersson for his contributions to the project, it is a pleasure to work together. Thank you to Kjell-Ove Mickelsson and Robert Johansson for their generous provisions of time, energy and invaluable industry advice. And finally thank you to my family, Cathy, Calvin and Maya for embarking on this journey with me.

\section{Mathematical Morphology Definitions}

The reader is referred to the following sources [6, 3] for thorough definitions of morphological erosion and dilation for $3 \mathrm{D}$ surface data and greyscale image processing. Using erosion and dilation the following simple explanations of some of the operations used in this research are provided. 
3D surface set

structuring element

erosion of set A by B

dilation of set A by $B$

closing of set A by B

opening of set A by B

black tophat

morphological laplacian

$$
\begin{aligned}
& A=\left\{(x, y, z) \in R^{3}\right\} \\
& B \text { (e.g. a small sphere of circle) } \\
& \epsilon_{B}(A) \\
& \delta_{B}(A) \\
& c_{B}(A)=\epsilon_{B}\left(\delta_{B}(A)\right) \\
& o_{B}(A)=\delta_{B}\left(\epsilon_{B}(A)\right) \\
& c_{B}(A)-A \\
& \left(\delta_{B}(A)-A\right)-\left(A-\epsilon_{B}(A)\right)
\end{aligned}
$$

\section{References}

[1] 3d scanner: Wikipedia, the free encyclopedia. http://en.wikipedia.org/wiki/3d_scanner.

[2] S. Beucher and F. Meyer. Mathematical Morphology in Image Processing, chapter 12, pages 433-481. Marcel Dekker Inc., 1992.

[3] E. R. Dougherty and R. A. Lotufo. Hands-On Morphological Image Processing. SPIE - The International Society for Optical Engineering, 2003.

[4] R. Ihaka and R. Gentleman. R: A language for data analysis and graphics. Journal of Computational and Graphical Statistics, 5(3):299-314, 1996.

[5] J. E. Larsson.info@mbvsystems.se.

[6] M. Thurley and K. Ng. Identifying, visualizing, and comparing regions in irregularly spaced $3 \mathrm{~d}$ surface data. Computer Vision and Image Understanding, 98(2):239-270, February 2005.

[7] M. Thurley and $\mathrm{K}$. Ng. Identification and sizing of the entirely visible rocks from segmented $3 d$ surface data of laboratory rock piles. In submission. Download from $\mathrm{http} / / /$ image3d6.eng.monash.edu.au.

[8] M. Thurley, K. Ng, and J. Minack. Mathematical morphology implemented for discrete, irregular $3 \mathrm{~d}$ surface data. In DICTA 99, Digital Image Computing: Techniques and Applications Conference Proceedings, Fifth Biennial Conference, pages 16-20, Curtin University, Perth, Western Australia, December 1999. Australian Pattern Recognition Society.

[9] M. J. Thurley. Three Dimensional Data Analysis for the Separation and Sizing of Rock Piles in Mining. PhD thesis, Monash University, December 2002. Download from http://image3d6.eng.monash.edu.au/thesis.html.

[10] W. Wang, F. Bergholm, and O. Stephansson. Image analysis of fragment size and shape. In J. Franklin and T. Katsabanis, editors, Measurement of Blast Fragmentation - Proceedings of the FRAGBLAST 5 Workshop, pages 233-243. Balkema, 1996. 